\title{
Implementación de un plan de manejo de fauna silvestre en la comunidad indígena Embera de Chageradó, en contexto de interculturalidad
}

\section{Implementation of a management plan of wild fauna in indigenous community Embera of Chageradó, in context of interculture}

\author{
Gloria Patricia Zuluaga ${ }^{1}$, Ubeimar Arango ${ }^{2}$, Liliana Castaño ${ }^{3}$
}

\section{RESUMEN}

El territorio de Chageradó, poblado por indígenas Embera, presenta una pérdida de valiosas especies animales debido a la explotación maderera. Esta investigación se realizó con el objetivo de restaurar el hábitat natural de la fauna silvestre ampliando la oferta de sus recursos alimenticios e integrando a ello la cultura. Se utilizó el método de Investigación-Acción-Participación que permitió la identificación de especies y la elaboración e implementación conjunta de un plan de manejo que incluyó el establecimiento de corredores biológicos, la instalación de saladeros y cebaderos, y la celebración de rituales jaibanísticos. Se logró identificar la importancia de las especies de fauna a nivel cultural, se identificaron y propagaron 14 especies vegetales para la construcción de corredores biológicos, 10 especies para los cebaderos y se realizaron cuatro ceremonias de retorno de las especies identificadas. Aunque todavía no es posible evaluar el impacto de todas las acciones, porque la restauración ecosistémica necesaria para el repoblamiento de la fauna silvestre requiere un proceso largo, se logró el aumento de áreas boscosas y de fauna silvestre, así como su adopción por parte de las comunidades.

Palabras clave: Fauna silvestre; Gestión ambiental en comunidades indigenas; Interculturalidad; Manejo ambiental.

\section{ABSTRACT}

The territory of Chageradó, populated by Embera natives, presents shortage of valuable animal species due to the lumber operation. Because recovering the natural habitat of the wild fauna extending the supply of its nutritional resources integrating to it the culture, this investigation was realized. It was used the method of investigation-participation-action that allowed to the identification of species and the elaboration and joint implementation of a handling plan that the establishment of biological corridors, the installation of salting hoses included and barley dealers, and the celebration of jaibanisticos rituals. It was managed to identify the importance of the species of fauna at cultural level, they identified and they propagated 14 vegetal species for the construction of biological corridors, 10 species for the barley dealers and 4 ceremonies of return of the identified species were realized. Although not yet it is possible to evaluate the impact of all the actions, since necessary the ecosystem restoration for the repopulation of the wild fauna requires a long process, was obtained the increase of wooded areas and wild fauna, as well as the adoption on the part of the communities.

Keywords: Wild fauna; Environmental management in indigenous communities; Intercultural; Environmental management.
1. Profesora Asociada, Universidad Nacional de Colombia, Bogotá, Colombia. e-mail:gpzuluag@unal.edu.co

2. Ingeniero Agrónomo, asesor de la Organización Indígena de Antioquia (OIA), Medellín, Colombia.

e-mail: egoronomia@hotmail.com

3. Bióloga, asesora de la Organización Indígena de Antioquia (OIA), Medellín, Colombia.e-mail:1iliana02@une.net.co Recibido: 4 de febrero de 2011 Aceptado: 3 de abril de 2011 


\section{INTRODUCCIÓN}

Las comunidades Embera que habitan los territorios del Atrato Medio antioqueño han experimentado fuertes transformaciones de su sistema alimentario debido a nuevos patrones de ocupación territorial y poblamiento; a cambios en el sistema de agricultura migratoria o itinerante, donde los ciclos de rotación de barbechos son cada vez más cortos, en unos suelos de vocación agrícola bastante limitada; a la explotación maderera a gran escala, que ha llevado, a su vez, al agotamiento de los recursos de caza, pesca y recolección, aspecto que se manifiesta en la escasez y desbalance de alimentos, tanto en calidad como en cantidad; y a la presencia del conflicto sociopolítico y armado que vive actualmente la región.

A finales de los años ochenta el territorio donde habita este grupo fue objeto de una actividad ilegal de explotación maderera que arrasó buena parte de la cubierta vegetal, por lo que sus formas de subsistencia, basadas en las actividades de cacería, recolección y pesca, se vieron seriamente comprometidas. Esta situación ha repercutido entre otros aspectos en la nutrición, la salud y las prácticas culturales de los indígenas. Por ello, la Organización Indígena de Antioquia (OIA) diseñó y emprendió un plan general de restauración con el objeto de mitigar el daño y restaurar las condiciones ambientales del territorio, que además, repercutiera en el mejoramiento de la seguridad alimentaria de la población indígena, y potenciara la cultura y la organización local y regional.

Este artículo describe y analiza el plan de manejo de fauna silvestre puesto en marcha en esta comunidad indígena en el período 2001-2003, resaltando el proceso intercultural en el cual se desarrolló.

\section{EL ÁREA DE ESTUDIO Y LA POBLACIÓN}

El Medio Atrato se caracteriza por una alta humedad, con precipitaciones entre $5000 \mathrm{~mm}$ y $9500 \mathrm{~mm}$ al año y una humedad relativa promedia del $80 \%$, que le confiere unas formaciones vegetales características de bosque muy húmedo tropical (bmh-T) y bosque pluvial premontano (bp-PM). Los suelos se encuentran principalmente Entisoles e Inceptisoles, con $\mathrm{pH}$ ácidos $(4,2$ a 5,2) debido a la alta lixiviación de nutrientes y a los bajos contenidos de materia orgánica por suacelerada mineralización(CODECHOCÓ 1988), condiciones que imponen fuertes restricciones de fertilidad para las actividades agropecuarias convencionales. En la Figura 1 se puede apreciar la ubicación de la zona de estudio.

Los Embera se encuentran dispersos en las selvas húmedas del Pacífico sumando, en conjunto, alrededor de 60.000 personas, que representan el $10,1 \%$ del total de la población indígena del territorio nacional (Salazar 2000). Su patrón de

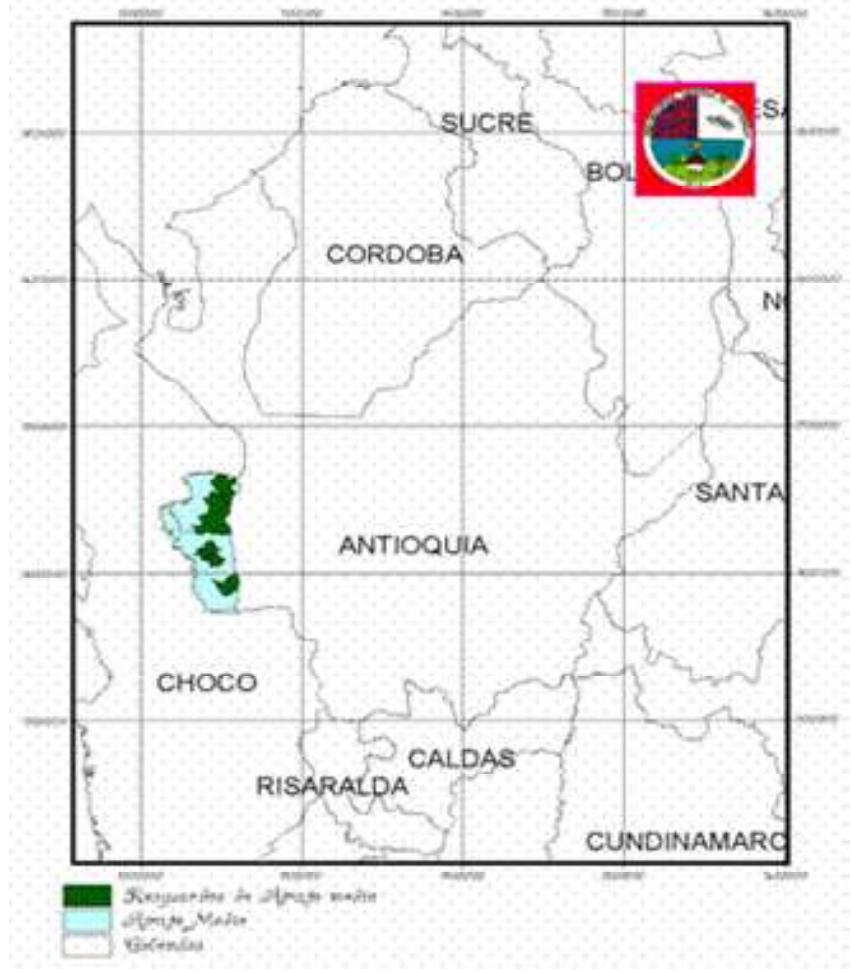

\section{Figura 1. Ubicación geográfica del área} de estudio.

Fuente: OIA

asentamiento está asociado, en las partes altas o medias de las cuencas tributarias del río Atrato, a los ríos que atraviesan sus territorios, donde establecen claros en la selva, por medio de la tumba, roza y pudre, ya que la alta humedad no permita realizar quemas, por lo tanto, el material de la tumba se dispone en el suelo para su descomposición, como una forma de abono verde. Cultivan principalmente plátano y maíz, complementado con gran diversidad de frutales, plantas medicinales y condimentos, alrededor de sus espacios habitacionales, en un arreglo que se ha denominado huertos mixtos tropicales, los cuales se clasifican como sistemas agroforestales simultáneos. Además, practican la recolección, la cacería y la pesca (Arango y Peñarete 2000, Betancur y Zuluaga 1988).

A pesar de mantener buena parte de sus tradiciones, los Embera vienen experimentando cambios en su cultura debido a situaciones como la pérdida de territorios ancestrales por presión colonizadora y la explotación maderera, que provocan el agotamiento de los recursos naturales; la incorporación de patrones alimentarios externos, que inducen la subvaloración de ciertos alimentos y técnicas agrícolas propias; los nuevos patrones de ocupación del territorio, resultantes de la constitución de resguardos con límites claramente establecidos; y la escasez de suelos con aptitud de uso agrícola y el conflicto armado, que no permite movilizarse a otros sitios del resguardo. 


\section{Bioetnia Volumen 8 No 1 (enero-junio), 2011}

A mediados de la década de los ochenta y principios de los noventa estas comunidades indígenas empezaron a experimentar una serie de procesos, casi simultáneos, en lo referente a la formación y construcción de cabildos, titulación y conformación de resguardos y fundación de poblados. En estas iniciativas han participado un sinnúmero de instituciones, que van desde las estatales, las religiosas, las de cooperación internacional y el mismo movimiento indígena, a través de la OIA. Las diferentes intervenciones se han enmarcado en tres líneas principales: la primera, dirigida al fortalecimiento organizativo; la segunda, encaminada a buscar seguridad alimentaria; y la tercera, enfocada al conocimiento y manejo del territorio y los recursos naturales. Las instituciones participantes han dirigido sus proyectos con una serie de modelos y enfoques que van desde ayudas puntuales de corte asistencialista, como también mediante programas bajo el nombre de proyectos de desarrollo rural, de desarrollo local, de desarrollo alternativo o incluso de desarrollo sostenible. Sin embargo, en la mayoría de los casos, no se han obtenido los objetivos planteados y han llegado a convertirse en parte de una problemática mayor donde, a nombre del desarrollo, la gente es más pobre cada día, en lo económico, social, cultural y político(Salazar 2000).

Por ello, la OIA se dio a la tarea de repensar y reconstruir la noción, tanto teórica como práctica, del desarrollo, proponiendo los planes de vida como una alternativa de planeación y gestión multidimencional, intercultural y participativa; esto es, involucrando aspectos sociopolíticos, culturales, económicos y ambientales, tendientes a mejorar la vida de las comunidades indígenas. Dentro de este nuevo marco de referencia se construye y se pone en marcha un plan de manejo de la fauna silvestre, que se desarrolló durante el período 2001-2003 en la comunidad indígena de Chageradó. Su objetivo fue llevar a cabo acciones y estrategias tendientes a restaurar el hábitat natural de la fauna silvestre ampliando la oferta de los recursos alimentarios consumidos por los animales. Para ello se elaboró, conjuntamente con los indígenas, una propuesta con tres componentes a desarrollar de manera simultánea: dos de ellos de carácter técnico y el tercero eminentemente cultural, que se describen a continuación de forma esquemática.

\section{METODOLOGÍA}

Para el levantamiento de información relacionada con las técnicas de caza de las comunidades indígenas de Chageradó y la relación de las especies silvestres con sus actividades culturales, se realizaron entrevistas con sabios y cazadores de la zona. Lo anterior permitió incluir este elemento dentro de los análisis requeridos para el diseño e implementación del plan de manejo de fauna silvestre. Estas entrevistas se emplearon también para identificar las especies vegetales más importan- tes y las principales causas de disminución de las poblaciones de fauna silvestre.

Dentro del proceso de diseño e implementación del plan de manejo, entendido como un contexto eminentemente intercultural, se buscó explorar concepciones y metodologías que permitieran la relación e interacción entre el saber de los indígenas y el saber de los técnicos, haciendo de ello un proceso investigativo inscrito dentro de la Investigación Acción Participativa (IAP), donde el «objeto a investigar», se considera sujeto de la misma, no solo para efectos de la investigación sino para conocer la realidad y transformarla.

El diseño del plan de manejo de la fauna silvestre se realizó con la participación de las comunidades indígenas de la región, mediante la realización de reuniones y talleres, se analizó la información levantada en campo y la información secundaria acerca de la zona. La propuesta lograda incluyó tres elementos fundamentales que son el establecimiento de corredores biológicos, la instalación de saladeros y cebaderos, y la celebración de rituales jaibanísticos.

Corredores biológicos. Para lograr el objetivo de generar conectividad entre los parches de bosque (de distintos estados sucesionales), a partir de sistemas agroforestales, parcelas de cultivos, rastrojos y parcelas de enriquecimiento forestal, se realizó una selección de las especies conjuntamente entre el equipo técnico y los miembros de la comunidad indígena, privilegiando aquellas espacies más afectadas por la explotación maderera, que fueran de mayor demanda por parte de la comunidad, y que adicionalmente, fueran favorables para el desarrollo de la fauna por su oferta de alimento y albergue.

La propagación y reproducción del material vegetal se realizó mediante el sistema de construcción de viveros, propuesta que involucró una serie de prácticas silvícolas como el trazado rectangular con cinta y brújula (Figura 2), una técnica que resultó difícil de asimilar por parte de los Embera, a pesar de que ya habían recibido capacitación previa.

Instalación de saladeros y cebaderos. Para el establecimiento y distribución espacial se tuvo en cuenta el conocimiento que tienen los cazadores sobre los sitios por donde se mueve la fauna, así como la observación de huellas (tipos de huellas y especies que las producen). Adicionalmente, se instalaron algunos saladeros en áreas donde se detectaron huellas de animales de caza, aunque no estuvieran dentro de las áreas delimitadas tradicionalmente para esta actividad.

El suministro de sal tenía como fin principal proporcionar elementos nutricionales necesarios para mejorar tanto el crecimiento como la función reproductiva de la fauna silvestre, específicamente de animales terrestres. El diseño planteado consistió en colgar de un árbol un bulto de sal de 50 kilos de peso, a una altura de cerca de 1,5 metros del suelo y un balde enterrado a ras del piso, exactamente debajo del saco de sal para recolectar la salmuera generada por efecto de la lluvia, de 


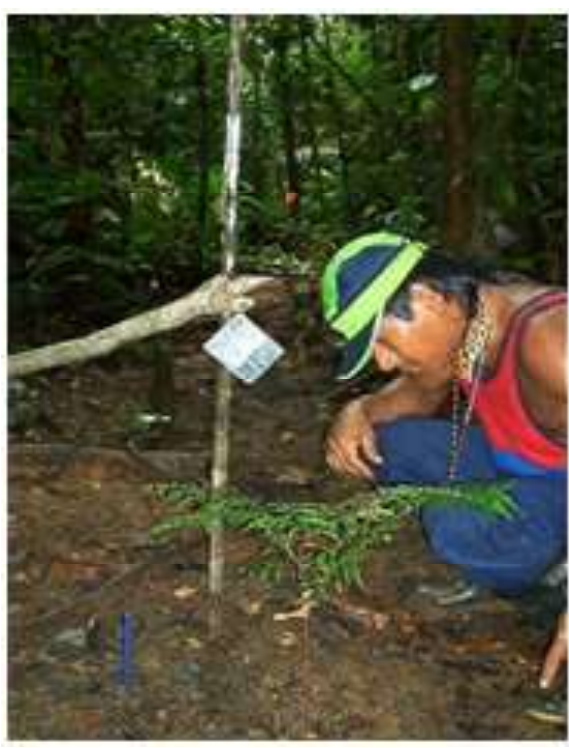

Figura 2. Fomento de parcelas de enriquecimiento forestal en biocorredores.

Fotografía Álvaro Agudelo

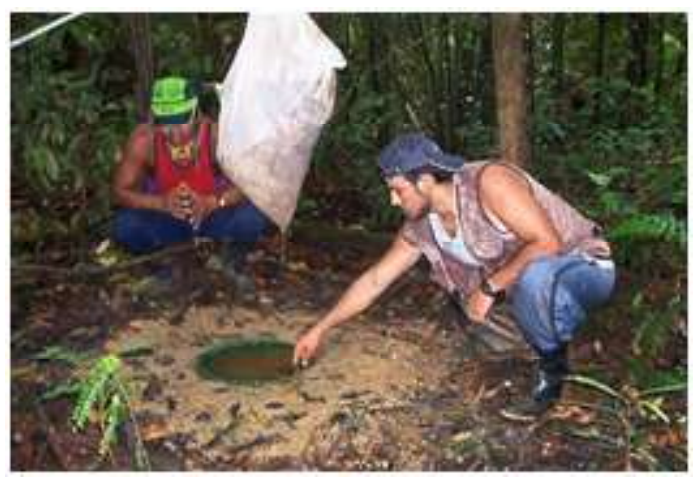

Figura 3. Instalación de cebaderos.

manera que se acumule y pueda ser consumida por los animales. Alrededor del balde se aplicó una capa de arcilla con el fin de obtener un registro de las huellas de los visitantes que permitieran su identificación posterior (Figura 3).

En relación con los cebaderos, estos consistieron básicamente en parcelas donde se estableció una combinación de cultivos transitorios (maíz, plátano, yuca) complementados con especies perennes (árboles frutales y palmas), sembrados de manera simultánea para que los frutales y palmas se desarrollaran mientras que los cultivos transitorios cumplían su ciclo vegetativo; de esta forma se garantizaba la oferta de frutos en diferentes momentos, convirtiendo el sitio en un atractivo importante para la fauna como fuente de alimento. Las parcelas establecidas tenían un área de $150 \mathrm{~m}^{2}$ (15 m x 10 $\mathrm{m})$, tamaño considerado como suficiente para atraer y alimentar la fauna silvestre.

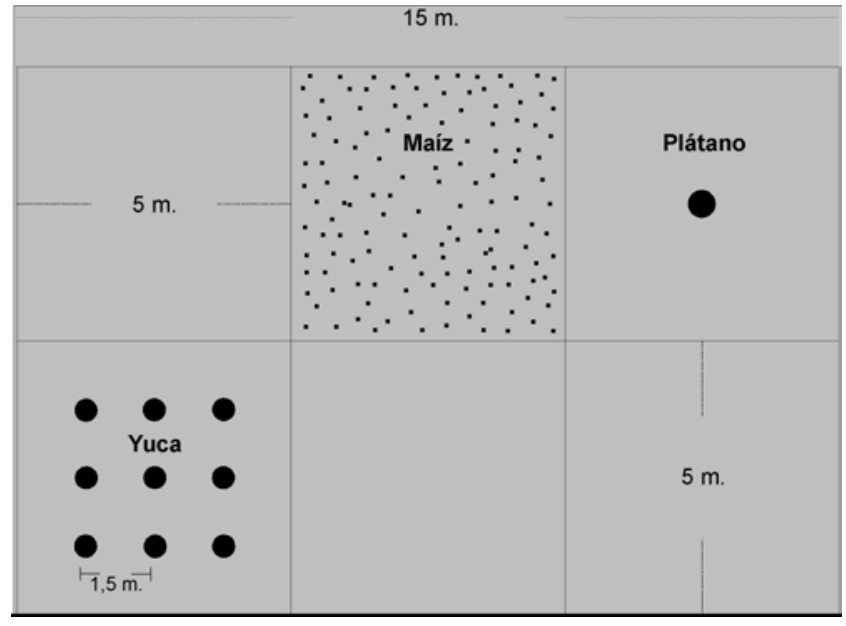

Figura 4. Esquema del diseño de los cebaderos.

En los cebaderos se distribuyeron árboles frutales y palmas, a una distancia promedio de cinco metros, hasta completar unos 12 individuos por parcela, dejando los cultivos transitorios al interior de los cuadros demarcados por las especies de frutales y palmas. Si el cultivo transitorio era yuca el distanciamiento fue de 1,5 m. El maíz se sembró al voleo y el plátano en el centro del recuadro de $25 \mathrm{~m}^{2}$ que crean los árboles y palmas. Para una mejor ilustración del diseño de los cebaderos (Figura 4).

Celebración de rituales jaibanísticos como medida de manejo, regulación y repoblamiento de fauna silvestre. Se organizaron y llevaron a cabo cuatro ceremonias periódicas para el retorno y repoblamiento del saíno, tatabro y guagua, con lo que se buscó una apropiación del plan por parte de las comunidades.

\section{RESULTADOS}

La cacería en las comunidades Embera. Se encontró que la caza es una de las prácticas tradicionales que identifican a los Embera de selva, siendo una actividad que recae principalmente sobre los hombres, porque representa uno de los mayores ideales masculinos. Es común entre los pueblos indígenas que los mejores cazadores reciban reconocimiento público, casi siempre en un contexto ritual, porque es señal de que tienen habilidades especiales. Alcaraz et al. (1988) señalan lo siguiente:

«En el cazar o 'montear' se evidencian las cualidades del hombre Embera. Quien debe conocer el comportamiento de los animales, dominar la técnica de elaboración de los miméticos olorosos con los cuales enjuaga a sus perros o se frota sus propias extremidades y además debe derrochar destreza y vigor físico persiguiendo a la presa por riscos y cañadas.»

El rol social de los cazadores tradicionales involucra muchos aspectos de la existencia y la vida de las comunidades 


\section{Bioetnia Volumen 8 No 1 (enero-junio), 2011}

tradicionales, porque se perciben como protectores de la comunidad contra todas las fuerzas del mal, como sabios y curanderos, y como aquellos que proporcionan las proteínas animales necesarias para el grupo humano (Fairhead y Leach 2002).

En lo que respecta a los usos, los Embera utilizan la mayoría de las especies como fuente de alimentación para satisfacer las necesidades de proteína de la familia, aunque en algunos casos se observa la venta interna de carne de monte con fines económicos, sobre todo cuando se trata de animales grandes como el venado, el sahino y el tatabro.

Entre los Embera de selva la cacería es la principal fuente de proteína animal, seguida por la proteína de animales domésticos (como gallinas y cerdos que se incorporaron a la dieta en la época de la Colonia). A nuestro parecer estas percepciones y prácticas en relación con la fauna silvestre y doméstica, se deben considerar cuando se desarrollen proyectos de producción animal en estas comunidades.

Para el caso de la Amazonia ecuatoriana, Descola (2005) describe lo siguiente: «la experiencia empírica del adiestramiento no ha conducido a los indígenas de la selva a procurar una verdadera domesticación, esto es, la reproducción controlada por el hombre de ciertas especies. Las razones son sin duda más culturales que técnicas. Ciertas especies autóctonas ocasionalmente adiestradas, como el pecari (concebidos por muchos grupos indigenas como cerdos domesticados colocados bajo la férula de amos sobrenaturales), probablemente habrían terminado por prestarse a una cría en semicautividad, proveyendo asi el equivalente de un cerdo. Pero tal robinsoneada hubiera sido utopía, porque si la caza exige el adiestramiento como su prolongación simbólica, la domesticación es su negación. La cría exige una relación de sujeción recíproca con el animal, viendo cada uno en el otro la fuente prosaica de su alimentación y su bienestar. Esta dependencia mutua adopta la máscara de una convivencia sin sorpresas en las antípodas del fervor lúdico que anima al cazador indio en la satisfacción cotidianamente renovada de su placer. La ausencia de animales domésticos locales parece menos atribuible a una falta de competencia que al hecho de que los amerindios son grandes experimentadores de lo viviente, atentos a sus propiedades múltiples y viejos maestros en los trabajos prácticos de genética vegetal».

Las técnicas de cacería que emplean los indígenas de Chageradó han sufrido grandes cambios en los últimos años; por ejemplo, de los tradicionales arco, lanza o flecha envenenada y bodoquera (cerbatana), han pasado a la utilización del perro cazador y de la escopeta. Sin embargo, el uso de esta última se ha visto restringido recientemente por las difíciles condiciones de orden público, al dificultarse su consecución, lo que ha llevado a construir unos artefactos que constan de tubos de hierro con pólvora, dotados de una especie de culata de madera, que los asemeja a un rifle y que denominan chispún.

Para los Embera la fauna no es solo fuente de alimento porque los animales, como poseedores de jais (espíritus), presentan una gama variada de usos dados por aspectos de tipo simbólico, como el prestigio, la protección, la medicina, la premonición y el ritual mediante actividades jaibanísticas, donde el Jaibaná (médico tradicional o Chamán, encargado de las relaciones con el mundo sobrenatural) determina el acceso o restricción en el uso del territorio y por ende de los animales. Además, las partes de los animales son importantes en la elaboración de adornos, tambores y mascotas.

Cuando se caza accidentalmente la madre de uno de estos animales (principalmente el loro, guacamaya, titigris, mico negro, guagua y ñeque), los Embera proceden a «adoptar»a las crías como mascotas, según comentan en una de las entrevistas «si quedan huérfanos hay que protegerlos, darles comida, porque no se pueden valer por sí mismos y se pueden morir... son como niños, por eso los llevamos pa' casa de nosotros». En este comentario se puede comprobar que existe una ética y unos conocimientos que están vinculados a la obligación de prestar ayuda, de cuidar o por lo menos el deber de no agresión a los animales. Nakashima y Roué (2002), hablando de los Cri de Canadá, mencionan que en las comunidades cazadoras existe una ética centrada en la visión de los animales como seres vivos, que engendra el principio de la responsabilidad de los hombres para con los animales. Presentan así un paralelismo entre la experiencia humana y la animal, haciendo hincapié en su índole similar, siendo este uno de los elementos fundamentales de la visión del mundo de estos indígenas. Para los Cri, los animales forman parte de un universo «sociocósmico» del que ellos mismos son un elemento integrante, los dioses les dieron a los autóctonos ese universo para que sacaran provecho de él y le prodigasen cuidados.

En lo referente al papel que cumplen los animales en la cosmogonía de los Embera, Ulloa etal. (1996), comentan que en las comunidades indígenas del Parque Nacional de Utría, utilizan para prácticas rituales la ombligada (práctica mágicoreligiosa, que se realiza para conferir poder o aumentar cierta capacidad en la persona, ejercida por el Jaibaná), que tiene como fin otorgarle a una persona las cualidades de un animal; así la raspadura de los huesos de la garra del oso hormiguero se usa para adquirir fuerza en los brazos y para pelear bien; los huesos del perezoso para trepar; los del armadillo para pelear; los del gato solo, el cusumbí y el tigrillo para dar fuerza en general; los del venado y el ñeque para correr; los de la zorra de agua para pescar y los de la marmota para andar de noche. Continúan diciendo las mismas autoras, que existen restricciones rituales sobre el consumo de algunos animales en ciertos momentos. Así, las mujeres que acaban de dar a luz no pueden consumir guagua y sahino, pero deben consumir 
ñeque. De igual manera, durante la primera menstruación, a la mujer se le recomienda consumir venado. Cada animal establece una relación integral al usar y apreciar sus partes; es así como el cráneo de un animal se utiliza para colgarlo del techo del tambo, lo que da prestigio al dueño de la casa, los sesos para preparar amuletos, la sangre para curar y los parásitos del animal como consejeros, ya que los Embera consideran que los parásitos pueden ayudar al cazador a atraer a la presa y el vuelo de un ave presagia muerte o vida.

Como se puede apreciar, la fauna silvestre ocupa una posición fundamental en el desarrollo de los grupos indígenas, tanto en términos simbólicos como materiales, por lo que es importante considerarla como un elemento determinante en los planes de vida, dado que es un recurso necesario para el ejercicio de sus derechos culturales y espirituales.

El plan de manejo de fauna silvestre. Entre los años 1987 a 1991, en la comunidad de Chageradó se realizó por parte de Maderas del Darién(MADARIÉN), una explotación ilegal de madera mediante tala rasa mecanizada del bosque, en una extensión aproximada de 7200 hectáreas, que ocasionó, entre otras problemáticas, la escasez y pérdida de valiosas especies animales de importancia alimenticia, tales como la guagua (Agouti paca), el tatabro (Tayassu tajacu), el saíno o jabalí (Tayassu pecari) y el venado (Mazama americana). Con el fin de amortiguar y mitigar los impactos de dicha explotación maderera se implementó de manera conjunta entre la OIA y las comunidades indígenas de la zona, un plan de manejo que incluyó los siguientes elementos:

Establecimiento de corredores biológicos. Uno de los propósitos fue el de generar conectividad entre los parches de bosque (de distintos estados sucesionales), a partir de sistemas agroforestales, parcelas de cultivos, rastrojos y parcelas de enriquecimiento forestal, cuyo conjunto formaba una especie de corredores biológicos entre los espacios de uso y manejo, esto teniendo en cuenta que muchos de los sistemas agrícolas, como las parcelas cultivadas, los huertos mixtos y los barbechos se convierten en fuentes de alimento para algunos animales silvestres e incluso son manejados para convertirlos en una especie de cebaderos. Un corredor biológico o biocorredor es una franja de vegetación, que busca facilitar los desplazamientos de los individuos de la fauna con el fin de extender las áreas de alimentación, reproducción y/o descanso. Se seleccionaron y propagaron especies vegetales con atributos multipropósito, de manera que desempeñaran diversas funciones ecológicas y culturales representadas en diferentes usos (Tabla 1). Para lograr este objetivo, la selección de las especies se realizó conjuntamente entre el equipo técnico y los miembros de la comunidad indígena, privilegiando aquellas especies más afectadas por la explotación maderera, que fueran de mayor demanda por parte de la comunidad y que adicionalmente, fueran favorables para el desarrollo de la fauna por su oferta de alimento $\mathrm{y}$ albergue.
Es importante destacar el conocimiento detallado que los indígenas poseen de los animales, en cuanto a sus hábitos alimenticios y de comportamiento, así como de sus variaciones estacionales. Este conocimiento indica que la fauna ha sido objeto de una observación y clasificación minuciosa, lo que se refleja además en un vocabulario especializado que da cuenta de un conocimiento ecológico local de gran importancia, que fue determinante para el éxito del plan. El hecho de que cada una de estas especies (animales y vegetales) tenga nombre en lengua Embera, habla de su cultura y del peligro que corre esta con la desaparición de los hábitats naturales y la desaparición de las actividades de cacería. Por ello, el proyecto buscaba potenciar elementos tangibles como fauna, árboles, alimentos, que a su vez permiten fortalecer intangibles, incluyendo algunas condiciones que hacen la vida posible. Resumiendo, el proyecto consideró que la fauna es un recurso necesario para el ejercicio de los derechos nutricionales, culturales y de culto.

Una vez realizada la propagación y reproducción del material vegetal mediante la construcción de viveros, se observó un alto grado de resistencia, que se tradujo en bajos niveles de apropiación y adopción, dada la concepción de los Embera de selva de que «los árboles en bosque nacen solos, nadie los siembra, ni tampoco crecen en fila y tampoco necesitan ser cuidados y manejados como los que están cerca de los tambos». El hecho de que estos indígenas no le vean sentido "a sembrar árboles en el bosque», hizo que se generara una continua tensión entre tradición y renovación del conocimiento, lo que plantea un reto de diálogo intercultural, así como la negociación entre prácticas y saberes de las diferentes formas de conocimiento.

Instalación de saladeros y cebaderos. Para el establecimiento y distribución espacial se tuvo en cuenta el conocimiento que tienen los cazadores sobre los sitios por donde se mueve la fauna, así como la observación de huellas (tipos de huellas y especies que las producen). Asimismo, se instalaron algunos saladeros en áreas donde se detectaron huellas de animales de caza, aunque no estuvieran dentro de las áreas delimitadas tradicionalmente para esta actividad.

El suministro de sal tenía como fin principal proporcionar elementos nutricionales necesarios para mejorar tanto el crecimiento como la función reproductiva de la fauna silvestre, en especial de animales terrestres. Mediante la técnica implementada se tuvo evidencia sobre todo de la presencia de saíno (Tayassu tajacu), guagua (Agouti paca) y venado (Mazama americana).

Sin embargo, el suministro de sal no contó con la suficiente acogida por parte de los indígenas, porque debido a la situación de orden público en la zona, los actores armados ejercen un estricto control sobre la distribución de los alimentos, dentro de los cuales la sal resultó ser uno de los más importantes. Para la población era casi incomprensible que un 


\section{Bioetnia Volumen 8 No 1 (enero-junio), 2011}

Tabla 1

Especies escogidas para los corredores biológicos

\begin{tabular}{|c|c|c|c|}
\hline Nombre científico & Nombre común & Nombre Embera & Usos \\
\hline Cariniana pyriformis (Lecytidaceae) & Abarco & Chibugá & $\begin{array}{l}\text { Construcción de vivienda y de } \\
\text { botes. } \\
\text { Construcción de champas }{ }^{1} \\
\text { Culatas de chispún (Escopeta) } \\
\text { Sogas o lazos } \\
\text { Alimento de fauna silvestre }\end{array}$ \\
\hline Virola flexuosa (Myristicaceae) & $\begin{array}{l}\text { Sangre de gallo, } \\
\text { virola }\end{array}$ & $\begin{array}{l}\text { Uruga, } \\
\text { nuanamo }\end{array}$ & $\begin{array}{l}\text { Maderable } \\
\text { Alimento para fauna silvestre }\end{array}$ \\
\hline Brosimun utile (Moraceae) & Sande, lechero & Murí & $\begin{array}{l}\text { Maderable } \\
\text { Construcción de champas } \\
\text { Alimento para la fauna silvestre }\end{array}$ \\
\hline Carapa guianensis (Meliaceae) & Guino & Wino & $\begin{array}{l}\text { Construcción de vivienda (tablas, } \\
\text { listones) } \\
\text { Alimento para la fauna silvestre }\end{array}$ \\
\hline Lacmellea sp (Apocynaceae) & Mora & Copidijo & $\begin{array}{l}\text { Construcción de canoas }{ }^{2} \\
\text { Bancas (maderable) } \\
\text { Alimento para la fauna silvestre }\end{array}$ \\
\hline Nectandra sp (Lauraceae) & Caidita & Caidita & $\begin{array}{l}\text { Construcción de champas } \\
\text { Alimento para la fauna silvestre }\end{array}$ \\
\hline Oenocarpus bataua (Arecaceae) & Palma mil pesos & Uruchira & $\begin{array}{l}\text { Provisión de aceite y alimentación } \\
\text { humana y de fauna silvestre }\end{array}$ \\
\hline Hymenaea courbaril (Caesalpiniaceae) & Algarrobo & & $\begin{array}{l}\text { Alimentación humana y de fauna } \\
\text { silvestre } \\
\text { Fijación biológica de nitrógeno }\end{array}$ \\
\hline $\begin{array}{l}\text { Astrocaryum standleyanum } \\
\text { (Arecaceae) }\end{array}$ & Wérregue & Jiwua & $\begin{array}{l}\text { Construcción de vivienda } \\
\text { Alimento fauna silvestre }\end{array}$ \\
\hline Catoblastos radiatus (Arecaceae) & Palma zancona & & $\begin{array}{l}\text { Construcción de vivienda } \\
\text { Alimento fauna silvestre }\end{array}$ \\
\hline
\end{tabular}

1. Nombre que reciben las canoas en la zona. 2. Nombre local usado para las canoas.

Fuente: Elaborado a partir de información de los indígenas

elemento tan escaso e importante se destinara a los animales del monte y se negaban sistemáticamente a suministrarla. Con el fin de disminuir la resistencia a dicha práctica, se les explicó que la sal es fundamental para la fauna porque les permite desarrollar sus procesos metabólicos y reproductivos, así como aumentar el porte, tamaño y peso. A pesar de ello, varios de estos saladeros fueron robados y algunos indígenas continuaron expresando "es lástima gastar sal en animales cuando la gente la carece...». Es importante anotar que la interculturalidad y el reconocimiento del otro no siempre suceden en completa armonía, sino que suponen también conflictos entre las diferentes formas de conocer y de entender el mundo.

En relación con los cebaderos con especies vegetales, se observó que fue de gran aceptación y adopción por parte de los Embera, quienes propusieron las especies vegetales a utilizar con base en su conocimiento sobre los hábitos alimenticios de la fauna. Las especies seleccionadas fueron aquellas que son consumidas total o parcialmente por animales como el venado, la guagua, el tatabro y el saíno. Además, se consideraron algunas estrategias de manejo de los espacios de uso agrícola y de sucesión del bosque, practicadas por los 
Tabla 2

Especies vegetales propuestas para los cebaderos

\begin{tabular}{|c|c|c|c|c|}
\hline Nombre científico & Nombre común & Tipo de cultivo & Nombre Embera & Fauna favorecida \\
\hline Zea mays & Maíz & Transitorio & Bee & $\begin{array}{l}\text { Guagua, paletón, oso } \\
\text { perezoso, guayacán }\end{array}$ \\
\hline Artocarpus altilis & Árbol del pan & Permanente & Wapana & Guagua, mico, ñeque \\
\hline Chrysophylum sp & Caimito & Permanente & Nesarrajo & $\begin{array}{l}\text { Guagua, paletón, mico } \\
\text { cariblanco }\end{array}$ \\
\hline Bactrys gacipae & Chontaduro & Permanente & Jengá & Guagua, venado, tatabro \\
\hline $\begin{array}{l}\text { Astrocaryum } \\
\text { standleyanum }\end{array}$ & Wérregue & Permanente & Jiwua & $\begin{array}{l}\text { Guacamayo, ardilla, ñeque } \\
\text { saino, tatabro, oso perezoso }\end{array}$ \\
\hline Inga sp & Guamo & Permanente & Tuetajó & $\begin{array}{l}\text { Guagua, mico cariblanco, mico } \\
\text { negro, perezoso }\end{array}$ \\
\hline Oenocarpus batava & Mil pesos & Permanente & Uruchira & $\begin{array}{l}\text { Ardilla, ñeque, loro, saino, } \\
\text { paletón, mico cariblanco, mico } \\
\text { negro, pava }\end{array}$ \\
\hline Clusiasp & Madroño & Permanente & $\begin{array}{l}\text { Sin denominación } \\
\text { Embera }\end{array}$ & Pavón \\
\hline Manihot esculenta & Yuca & Transitorio & $\begin{array}{l}\text { Sin denominación } \\
\text { Embera }\end{array}$ & Guagua, tatabro, ñeque \\
\hline Musaceas & Banano, plátano & Transitorio & Patá en general & $\begin{array}{l}\text { Gagua, diferentes especies de } \\
\text { aves }\end{array}$ \\
\hline Persea americana & Aguacate & Permanente & Vego & $\begin{array}{l}\text { Ardilla, diferentes especies de } \\
\text { aves, guagua }\end{array}$ \\
\hline
\end{tabular}

Fuente: Elaborada a partir del conocimiento de los indígenas

Embera del Atrato Medio, que consisten en dejar plantas domesticadas y/o en estado silvestre dentro de las parcelas de cultivo con el fin de atraer animales de cacería hasta allí. Las especies seleccionadas se muestran en la Tabla 2.

La restauración de las condiciones ambientales para el repoblamiento de la fauna silvestre es un proceso de largo plazo; por tanto, aún no se cuenta con toda la información que permita evaluar su impacto. Sin embargo, a través de monitoreos, se pudieron observar hojas y frutos mordidos, así como la presencia de huellas, lo que se puede considerar como un «acierto» del plan de restauración. Infortunadamente, algunos de los indígenas capturaban los animales que encontraban en las parcelas, por lo que fue necesario realizar una serie de reuniones orientadas a recordarle a la comunidad los objetivos del plan. De esta manera, se concertaron y establecieron una serie de disposiciones, tales como un reglamento interno sobre el uso y manejo de los recursos naturales dentro del resguardo, que incluía no practicar cacería en los sitios donde se estaba implementando la propuesta y aclarar que solo en el largo plazo se podría incrementar el potencial productivo de la zona. Asimismo, y como contrapartida, el plan de manejo se comprometió a adelantar en forma paralela, un proyecto de producción de especies menores para mejorar la oferta de proteína animal, sin embargo, no se tuvo el éxito esperado.

Celebración de rituales jaibanísticos como medida de manejo, regulación y repoblamiento de fauna silvestre. Se encontró que para los Embera el espacio donde se reproducen y habitan los animales tiene una connotación espiritual que está regulada desde lo simbólico; dentro de su cosmogonía los animales pertenecen a otro mundo, al mundo de abajo, a diferencia del conocimiento académico, donde la vida ocupa un lugar meramente biofísico, por lo que se construye en una dualidad de pares en oposición naturaleza-cultura, dualidad que es a la vez conceptual y metodológica. Para los indígenas no existe un único ordenamiento de elementos de la naturaleza y sus sistemas de clasificación están formados por múltiples criterios con categorías que tienen varios significados. Para los indígenas la disponibilidad o ausencia de los animales de caza depende de la calidad del hábitat natural y del Jaibaná, 


\section{Bioetnia Volumen 8 No 1 (enero-junio), 2011}

dado que estos indígenas tienen el poder de conectar los dos mundos, y se cree que pueden manejar y controlar los Jais o espíritus de los animales.

La escasez del recurso, por temporadas no muy largas, está asociada a la «malevolencia» de un Jaibaná foráneo o ya desaparecido. Es así como para los indígenas de Chageradó la escasez de animales de monte está asociada a un Jaibaná de la comunidad de Bolsa (Comunidad indígena de la zona de estudio en Turriquitado-Llano), que tiene encerrada la casa donde moran los animales, principalmente el saíno y no los deja salir de los sitios sagrados; motivo por lo cual era necesario realizar unos rituales para «abrir las puertas de la selva a los animales del monte». Es importante mencionar que estos sitios sagrados coinciden con las zonas de alto interés ecológico dado que allí existen condiciones naturales excepcionales para la reproducción de la fauna silvestre.

Así nos lo cuenta José Joaquín Domicó, líder indígena de la zona: «Don Juaco, el Jaibaná de Bolsa, puede ir en sueños hasta las cabeceras de los ríos, alli tiene encerrado el saíno. No lo deja salir por envidia, pues el Jaibaná no puede matar al dueño de esos saínos, aunque le pegue un tiro no muere, no le entra el arpón [...] una vez llegó otro Jaibaná y empezó a matar a bastantes sainos y no le dio carne al Jaibaná de Bolsa. Por esto los saínos se fueron perdiendo. Don Juaco los encerró con un cerco. Algunos se lograron salir del cerco. Se deja un poquito afuera para que la gente coma. La gente empezó a matar bastantes y a vender, y al Jaibaná no le gustó eso y cerró. Al Jaibaná tampoco le gusta que coman solos, sino que le den parte de la carne a él. Don Joaquín ha dicho eso a su sobrino estando borracho. También dijo que si la comunidad se compromete a darle un saíno cuando cace, él se compromete a abrir, eso hicimos una vez yél abrió, le dimos una sola toma de benecuá (Bebida de maíz fermentada la cual es consumida en fiestas y en cierta clase de ritos ceremoniales); pero él pide cuatro fiestas para abrir. En la fiesta hay que darle tabacos, cigarrillos, aguardiente y chicha. Y darle fruto de barrigona (palma Iriatea ventricosa), o sea la comida de los animales. En sueños él va echando las frutas y los animales van comiendo hasta que salen afuera [...] cada animal tiene su jefe que maneja al grupo. Tatabro, saíno, mico, guagua; cada uno tiene jefe, un parcito, macho y hembra. Ahora casi no hay saíno, de vez en cuando se encuentra el rastro nada más. Don Joaco dizque abrió un grupo pequeño con una beba que le dimos». (Entrevista, agosto 28 de 2002).

Como vemos, existe la creencia de que la conducta, el desplazamiento y la reproducción de los animales, y por ende, el éxito en la caza, están condicionados por conductas y acontecimientos sociales, que conciernen al comportamiento doméstico de los habitantes del grupo y a sus relaciones personales pacíficas o conflictivas. Al romper estas reglas de comportamiento, ya sea deliberadamente o por accidente, se podrían «cerrar las puertas» del monte y para restablecer un orden socioecológico correcto se requiere que la tierra sea sometida a una «limpieza» por un especialista que conoce las leyes de los espíritus ancestrales de la zona.

En otro relato el mismo líder indígena dice: «Mi abuelo era Jaibaná. El montaba encima de Nusí para jugar. El Nusí es como una serpiente larga con alas verdes, lengua roja, se esconde en el agua, hay cinco clases de Nusí: Quicharo, Guabinacuruba, y Nusí de arena. Vive enterrado y se lo traga a uno para abajo y se lo come. El Nusí de pantano vive en cualquier laguito. El que se convierte en Nusí solo machuca y chupa sangre nada más. Mi abuelo materno era Jaibaná y le gustaba montar encima de ese caimán, de ese Nusí, mejor dicho le gustaba jugar con animales fieros de agua».

El considerar dentro del proyecto la percepción de los indígenas sobre la fauna hizo posible comprender y por lo tanto, vincular con el plan de manejo, el conocimiento del Jaibaná, y en general, las creencias de los Embera sobre la oferta de fauna; por ello se organizaron y llevaron a cabo cuatro ceremonias periódicas para el retorno y repoblamiento del saíno, tatabro y guagua; actividades estas que repercutieron positivamente en la apropiación del proyecto, y en el cumplimiento del reglamento. Tal como ponen de relieve Leach y Fairhead (2002), los conocimientos y las creencias sobre temas «técnicos» son en gran parte inseparables de la cosmología y de la religión local, por un lado, y de las cuestiones de orden social y de las relaciones predominantes de autoridad, por el otro.

Toledo (2005), comenta que para lograr una correcta comprensión de los saberes locales, se deben analizar las relaciones, tanto de las actividades prácticas como del sistema de creencias de la cultura o del grupo humano al que pertenecen. Así, la etnoecología integra en sus análisis el conjunto de creencias (COSMOS), el sistema de conocimientos (CORPUS) y el conjunto de prácticas productivas (PRAXIS), lo que permite comprender cabalmente las relaciones que se establecen entre la interpretación o lectura del uso, y manejo de la naturaleza y sus procesos.

Después de la celebración de las ceremonias (Figura 5), los Embera expresaban su preocupación por la presencia de culebras y de pisadas de tigre en estas zonas. Rafael Valencia, el Jaibaná de Chageradó, ya había explicado que cuando se está abriendo el camino del saíno y el venado es muy probable que salga el espíritu del tigre y la culebra, siendo difícil controlar su salida. Esta explicación tranquilizó a los Embera quienes ven en el Jaibaná una manifestación de dualidad entre el bien y el mal, pero en todo caso, sus opiniones son altamente respetadas.

Frente a la posibilidad de retorno y reproducción de los animales de monte, el Jaibaná Rafael expresó: «Yo abro la casa para que ellos salgan, pero la comunidad y ustedes los 


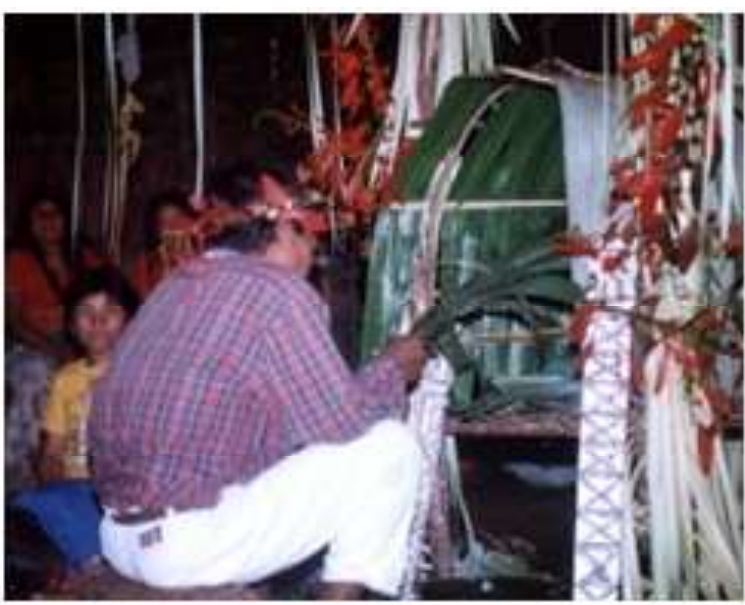

Figura 5. Ritual jaibanístico para el repoblamiento de fauna silvestre

Fotografía: Ubeimar Arango

técnicos deben de ayudar en su regulación y manejo, puesto que si por ejemplo empiezan a ser cazados de una manera ligera y indiscriminada y su carne a no ser compartida van a volver a ser encerrados por el Jaibaná Juaco». (Entrevista, julio 30 de 2002).

\section{CONCLUSIONES}

La evaluación de los impactos a nivel ecosistémico y productivo generados por el plan de manejo ambiental de fauna silvestre implementado en la comunidad de Chageradó, requieren de mayor tiempo para su análisis definitivo, pues la restauración ecosistémica necesaria para el repoblamiento de la fauna silvestre es un proceso a largo plazo. Sin embargo, este avance logró el aumento de áreas boscosas y de fauna silvestre, así como la adopción, apropiación y alto índice de participación por parte de la comunidad, por la inclusión del componente cultural dentro de la investigación.

La búsqueda de soluciones en la gestión de los proyectos de medio ambiente y desarrollo, debe darse en estrecha colaboración y diálogo con los actores afectados, lo que además de permitir un mayor éxito en las acciones, propicia un enriquecimiento de los métodos convencionales de la investigación y una nueva configuración de los saberes.

En la gestión y la puesta en marcha de proyectos de desarrollo en comunidades indígenas, se presenta una continua tensión entre tradición y renovación del conocimiento y de las prácticas productivas, de conservación y alimentarias. Estas comunidades no se han mantenido «libres» de la influencia de factores externos, y por tanto, no se mantienen en una tradición congelada. Ello plantea que la riqueza cultural de estos pueblos no es un instrumento suficiente para enfrentar las problemáticas y los retos de la sostenibilidad ambiental, social y política.

Para los indígenas de Chageradó el mundo de los animales constituye un complejo sistema donde interaccionan saberes y prácticas culturales, religiosas, tecnológicas, económicas y ecológicas de gran importancia para un uso y manejo sostenible del territorio. Sin embargo, no ha sido suficientemente considerado y valorado por el saber académico occidental y por tanto no se ha incorporado en los modelos de gestión ambiental de las instituciones formales.

El diseño y la gestión de proyectos de desarrollo, se da en una interacción explícita entre ciencia y sociedad, por lo que se hace necesario un enfoque de negociación participativa e intercultural entre actores y saberes, donde se presentan por igual conflictos y diálogos posibles entre diferentes formas de conocimiento.

\section{LITERATURA CITADA}

Alcaraz, G., Arias, M., Gálvez A. 1988. Situación de salud materno infantil en asentamientos Embera de Dabeiba, Antioquia. Informe Final. Proyecto de investigación. COLCIENCIAS, Universidad de Antioquia, CENICS. Dabeiba. 256 pp.

Alcaraz, G. 1996. Estado de salud de la población. Embera de Chajeradó. p. 14059. En: Chajeradó río de la caña flecha partida. Bogotá: Colcultura.

Arango, U., D. Peñarete. 2000. Estrategias de producción, extracción y protección en los territorios de las comunidades Embera de Jarapetó, Jengadó y Ñarangué (Medio Atrato antioqueño). Tesis Ingeniería Agronómica e Ingeniería Forestal. Medellín: Universidad Nacional de Colombia. 235 pp.

Betancur, C., G. Zuluaga. 1988. Aspectos agrotecnológicos de la comunidad Emberá en las veredas Chuscal y Tuguridó (Dabeiba, Antioquia). Seminario Ingeniería Agronómica, Universidad Nacional de Colombia, Sede Medellín. 106 pp.

Correa, F. 1993. La selva humanizada: ecología alternativa en el trópico húmedo colombiano. $2^{\mathrm{a}}$ ed. Bogotá: ICAN-Fondo FEN-Fondo Editorial CEREC.

Descola, P. 2005. Las lanzas del crepúsculo. Relatos Jíbaros alta Amazonia. Buenos Aires: Fondo de Cultura Económica. 450 pp.

Duque, L. 1997. Chageradó. El río de la caña flecha partida. Bogotá: Colcultura. $161 \mathrm{pp}$.

Fairhead, J., M. Leach. 2002. Modos de contestación: la «ciencia ciudadana» y los «conocimientos indígenas» en África occidental y el Caribe. Rev Internac Cienc Soc UNESCO. 173: 19-35.

Nakashima, D., M. Roué. 2002. Conocimientos tradicionales para evaluar las repercusiones del desarrollo moderno de tipo occidental en el medio ambiente. Rev Internac Cienc Soc UNESCO. 173: 62-77.

Organización Indígena de Antioquia. 2000. Plan de vida de las comunidades indigenas Embera Katio de Ibudó y Karrá en la región de Urabá, Antioquia. Medellín: OIA. 125 pp.

Organización Indígena de Antioquia. 1992. Plan de etnodesarrollo para las comunidades indígenas de Antioquia. Medellín: OIA. 190 pp.

Organización Indígena de Antioquia y ESPAVÉ. 1999. Experiencia piloto en ordenamiento territorial en el Resguardo de los Ríos Jengadó, Patadó. Medellín: OIA. 215 pp.

Organización Indígena de Antioquia. 2003. Plan de restauración y manejo ambiental de Chageradó fase III. Medellín: OIA. 212 pp.

Organización Indígena de Antioquia 2002. Plan de restauración y manejo ambiental de Chageradó fase II. Medellín: OIA. 280 pp.

Organización Indígena de Antioquia 2001. Plan de restauración y manejo ambiental de Chageradó fase I. Medellín: OIA. 315 pp.

Rojas, T. 2002. Plan de Vida. En: Palabras para desarmar. Una aproximación crítica al vocabulario del reconocimiento cultural en Colombia. Bogotá: Ministerio de Cultura. Instituto de Antropología e Historia. p. 341-50. 


\section{Bioetnia Volumen 8 No 1 (enero-junio), 2011}

Rist, S. 2002. Si estamos de buen corazón, siempre hay producción. Caminos en la renovación de formas de producción y vida tradicional y su importancia para el desarrollo sostenible. La Paz: Ed. Agruco. 508p.

Salazar, C. 2000. Dayi Drua nuestra tierra. Comunidad y territorio indigena en Antioquia. Medellín: Pregón Ltda. 69 pp.

Toledo, V. 2005. La memoria tradicional: la importancia agroecológica de los saberes locales. LEISA Rev Agroecol. 20 (4): 17

Toledo, V. J. Carabias, C. Mapes, C. Toledo. 1993. Ecología y autosuficiencia alimentaria. Hacia una opción basada en la diversidad biológica ecológica y cultural de México. Veracruz: Siglo XXI Editores. 118 pp.

Ulloa, A., Rubio, H., Campos, C. 1996. Trua Wandra: Estrategias para el manejo de la fauna con comunidades Embera en el parque Nacional Natural de Utría, Chocó, Colombia. Santafé de Bogotá: Fundación Natura OREWA/Ministerio del Medio Ambiente/OEI. 288 pp.

Villa, W., Valencia, E. 1992. Plan de etnodesarrollo para las comunidades indigenas de Antioquia. Propuestas y proyectos. Medellín: OIA. 137 pp 